\title{
Caracterização de Desempenho de uma Rede LoRa em Ambientes Urbanos: Simulação vs. Prática
}

\author{
Fernando M. Ortiz ${ }^{1}$, Thales T. Almeida ${ }^{1,2}$, \\ Ana Elisa Ferreira ${ }^{1,3}$ e Luís Henrique M. K. Costa ${ }^{1}$ \\ ${ }^{1}$ Universidade Federal do Rio de Janeiro - PEE-COPPE/GTA \\ ${ }^{2}$ Centro Federal de Educação Tecnológica de Minas Gerais - CEFET/MG \\ ${ }^{3}$ Centro Federal de Educação Tecnológica Celso Suckow da Fonseca - CEFET/RJ \\ \{fmolano, almeida, ferreira, luish\}@gta.ufrj.br
}

\begin{abstract}
LoRa, a low power wide area network technology, enables sensing in Smart Cities increasing coverage and reducing network infraestruture. Nevertheless, works about LoRa lacks simulation evaluations, being limited to experiments with a reduced number of physical devices. Because software developments of the LoRaWAN architecture are emerging, and the necessity to verify the equivalence between practical and simulated experiments, this work compares the results of experimentation using LoRa physical devices and simulations using NS-3 software. The results show that there is strong correlation between the metrics evaluated in each environment. Simulations using an adapted stochastic propagation model demonstrate that are similar to real experiments, with equivalent results for performance tests using the LoRa setup parameters.
\end{abstract}

Resumo. A LoRa, uma tecnologia de baixa potência e longo alcance, possibilita o sensoriamento em Cidades Inteligentes aumentando a cobertura, e reduzindo a infraestrutura da rede. No entanto, a literatura sobre a LoRa carece de avaliações de simulação, estando limitada à experimentação de um número reduzido de dispositivos físicos. Devido ao surgimento de implementações em software da arquitetura LoRaWAN, é necessário verificar a equivalência entre experimentos práticos e simulados. Com foco neste objetivo, este trabalho compara os resultados da experimentação usando dispositivos físicos LoRa e simulações usando o software NS-3. Os resultados mostram que existe uma forte correlação entre as métricas avaliadas em cada um dos ambientes. Simulações usando um modelo de propagação estocástico adaptado mostram que elas são semelhantes aos experimentos reais, com resultados equivalentes para todos os testes de desempenho, usando os parâmetros de configuração da LoRa.

\section{Introdução}

Dispositivos com capacidade de se conectar e se integrar têm permitido que múltiplas "coisas" tenham a capacidade de se comunicar através da Internet. A partir desse paradigma, tais dispositivos têm se tornado inteligentes, capazes de capturar, processar e transmitir dados. Estes dispositivos têm a capacidade de serem autônomos, permitindo, inclusive, que o enlace de comunicação entre eles seja gerenciado. Por isso, a quantidade de objetos inteligentes transmitindo informações na Internet é estimada em 
50 bilhões de dispositivos até 2020 [Evans, 2011]. Este enorme crescimento leva à necessidade de desenvolver novas tecnologias. As redes de baixa potência e longo alcance (Low Power Wide Area Network - LPWAN) como, por exemplo, a tecnologia LoRa (Long Range), são uma opção para atender a esta necessidade. Cidades inteligentes, smart grid e sensoriamento urbano são aplicações que ilustram bem este cenário com a comunicação de pontos distantes e que requerem baixo consumo de energia.

A LoRa é uma tecnologia de comunicação sem-fio, orientada a complementar as redes de telefonia móvel legadas e as tecnologias sem-fio de curto alcance. É indicada, principalmente, para aplicações de Internet das Coisas (Internet of Things - IoT) que requeiram cobertura de grandes áreas [Augustin et al., 2016, Raza et al., 2017]. Para isso, a LoRa implementa uma técnica de modulação por espalhamento espectral de chirps (Chirp Spreading Spectrum - CSS) [Semtech Corp., 2015], que varia a frequência sem mudar a fase entre símbolos adjacentes [Goursaud e Gorce, 2015], fazendo com que o sinal resultante seja resistente a interferências por ruído ou sinais com frequências próximas. A LoRa também se caracteriza pela redução na complexidade do hardware, a diminuição do tamanho dos cabeçalhos e da complexidade da rede em termos de saltos e endereçamento, viabilizando uma comunicação bidirecional simples, com o uso de uma infraestrutura mínima e com baixo consumo de energia. Entretanto, o planejamento de redes LPWAN torna-se complexo pelos múltiplos aspectos da sua implementação, que podem ser avaliadas por meio de simulações. Uma vez que as redes LPWAN utilizam radiofrequência como meio de transmissão, a variação das condições no meio de propagação, as limitações em termos de distância e interferência, além da utilização de um meio de comunicação compartilhado, fazem da simulação de redes LPWAN um desafio importante para avaliar o comportamento dessas e outras varáveis no ambiente simulado. Embora existam outras tecnologias LPWAN [Raza et al., 2017], este documento foca na tecnologia LoRa, por ser uma das mais difundidas atualmente.

Neste trabalho, é investigada a equivalência entre os resultados de simulações executadas no simulador de redes NS-3 e experimentos práticos que envolvem a comunicação de dispositivos reais compatíveis com a tecnologia LoRa. Para isso, é feita uma avaliação de desempenho da comunicação em enlaces compostos por uma unidade transmissora e uma unidade receptora, baseado na metodologia usada em [Ortiz et al., 2018], variando a distância e o fator de espalhamento (Spreading Factor - SF). São também implementados protótipos de hardware para a comunicação LoRa, onde as mesmas condições de simulação são recriadas. São avaliadas as seguintes métricas: capacidade da rede em termos de vazão, taxa de perda de mensagens, potência do sinal recebido e relação entre vazão teórica e a vazão obtida através dos experimentos. Para as simulações, utilizou-se uma biblioteca para redes LoRaWAN [Magrin et al., 2017], desenvolvida para o simulador de eventos discretos NS-3 (Network Simulator version 3) [Carneiro, 2010, Henderson et al., 2008].

Uma vez que as atuais tecnologias LPWAN (LoRa e SigFox) na camada física são proprietárias, poucas soluções de simulação têm sido desenvolvidas para modelar seu comportamento em diferentes ambientes com linha de visada (Line-of-Sight - LoS) ou sem linha de visada (Non-Line-of-Sight-NLoS). Assim, vários autores têm desenvolvido experimentos práticos avaliando as suas diversas características (especialmente da LoRa) em vez de simulação. Apesar deste crescente desenvolvimento de soluções práticas im- 
plementando a tecnologia LoRa, as variações nas condições do meio de propagação, o curto tempo de contato entre os nós, os problemas de atenuação e os fenômenos de multipercurso tornam estas implementações muito específicas e fazem necessária a avaliação desses comportamentos por meio de simulações que possam ser reproduzidas facilmente e em situações previamente determinadas. Além disso, experimentos de grande porte são mais difíceis de serem realizados no cenário real em comparação aos projetados em ambiente simulado. O tratamento dos dados gerados por simulação possibilita ainda uma melhor compreensão do desempenho de um enlace antes de ser implementado na prática e a identificação de um comportamento anômalo, antes da entrada em operação.

Este trabalho está organizado da seguinte forma. A Seção 2 discute os trabalhos relacionados com experimentos práticos e simulações com a tecnologia LoRa. A Seção 3 descreve a tecnologia LoRa, mostrando a camada física (LoRa PHY). Já a Seção 4 apresenta a metodologia utilizada neste trabalho, assim como os protótipos de hardware e software implementados para os experimentos. A Seção 5 apresenta os experimentos e resultados obtidos. Por fim, a Seção 6 conclui o trabalho e identifica desafios futuros.

\section{Trabalhos Relacionados}

Poucos trabalhos têm sido desenvolvidos com o intuito de caracterizar e avaliar o comportamento das redes LPWAN em um ambiente simulado. Bor et $a l$. [Bor et al., 2016b] desenvolveram uma ferramenta de simulação de eventos discretos chamada LoRaSim usando o arcabouço SimPy. Esta ferramenta permite alocar $N$ nós e $M$ gateways em um espaço bidimensional. Cada nó é definido por parâmetros de transmissão específicos da tecnologia LoRa. Assim, a ferramenta LoRaSim permite avaliar a quantidade máxima de dispositivos terminais em um enlace LoRa. Para estabelecer os parâmetros de alcance e captura de pacotes, os autores desenvolveram um modelo experimental prático. O alcance da comunicação foi determinado em função do $\mathrm{SF}$ e da largura de banda (Bandwidth - BW), e a captura de pacotes em função do tempo de transmissão e da potência do sinal. Os autores concluem que múltiplos sumidouros, bem como a configuração dinâmica dos dispositivos, permitem melhorar a escalabilidade na rede. Utilizando o trabalho de [Bor et al., 2016b], Centenaro et al. [Centenaro et al., 2016] investigam os efeitos da interferência em um gateway LoRa. Para isso, empregando a ferramenta LoRaSim, avaliam duas condições de interrupção em um enlace, uma baseada na relação sinal-ruído e a outra baseada na interferência entre sequências iguais do SF. Partindo dessas condições, os autores concluem que os enlaces são altamente sensíveis às colisões, o que reduz consideravelmente a escalabilidade da rede.

Reynders et al. [Reynders et al., 2016] avaliam as diferenças técnicas entre duas tecnologias sem-fio para redes LPWAN: LoRa e SigFox. Diferenciadas pelo tipo de modulação que usam, LoRa - espalhamento espectral por chirp, e SigFox - banda ultra estreita (Ultra Narrow Band - UNB), ambas as tecnologias podem operar na mesma frequência. Os autores avaliaram a coexistência das duas tecnologias na camada física. Para isso, implementam uma simulação em MATLAB para avaliar a taxa de erro de bit nas técnicas de modulação, CSS e BPSK (usada pela UNB). Já para avaliar a camada de controle de acesso ao meio, os autores comparam o desempenho baseado em interferência e coexistência. Para isso, Reynders et al. desenvolveram ambientes de simulação no simulador de eventos discretos NS-3. Para avaliar a interferência, foi projetado um cenário com uma área de $1000 \mathrm{~m} x 1000 \mathrm{~m}$ com um nó central. Foram simulados cenários avali- 
ando a interferência entre duas redes com modulação CSS, e um outro entre UNB e CSS. Os autores concluem que o UNB tem melhor desempenho que o CSS para grandes quantidades de nós a longa distância. Porém, o CSS oferece melhor desempenho em relação à vazão, e a variação do SF permite atingir maiores distâncias.

Magrin et al. [Magrin et al., 2017] avaliaram o desempenho de redes LoRa em um cenário de cidades inteligentes. Os autores propuseram uma medição para avaliar o desempenho de um enlace, implementando uma biblioteca LoRaWAN no NS-3. Os autores mostraram que o LoRaWAN fornece uma taxa de transferência mais alta do que o esquema básico do ALOHA, e que as redes LoRaWAN podem ser dimensionadas à medida que o número de gateways aumenta. Ao mesmo tempo, estudam o impacto do tráfego de downlink. Os autores concluem confirmando que os resultados de dimensionamento da rede são devidos à ortogonalidade entre os SFs. Também concluem que a arquitetura é facilmente escalável, ainda mais quando o número de gateways aumenta, melhorando a cobertura e a confiabilidade no enlace de uplink. Já Abeele et al. [Van den Abeele et al., 2017] implementaram uma biblioteca LoRaWAN no NS-3 que inclui um modelo de erro usado para determinar o alcance, assim como a interferência entre múltiplas transmissões simultâneas. Além disso, o módulo também suporta comunicação bidirecional. Os autores concluem que a configuração dinâmica de parâmetros nos dispositivos terminais é determinante para o desempenho da rede. Também concluem que o fluxo limitado do enlace de downlink degrada consideravelmente a taxa de entrega de pacotes, e que aumentando o número de gateways o problema pode ser mitigado. As camadas físicas LoRa PHY implementada tanto em [Magrin et al., 2017] quanto em [Van den Abeele et al., 2017] suportam todos os SFs e todas as taxas de código indicadas na especificação.

Petäjäjärvi et al. [Petäjäjärvi et al., 2015] avaliam uma tecnologia de baixo consumo de energia e longo alcance, implementando a tecnologia LoRa. Para isso, os autores fazem experimentos práticos para avaliar a cobertura em diferentes ambientes: (i) um ambiente aquático, no qual instalaram um dispositivo terminal transmitindo a partir de um barco no mar; e (ii) um ambiente terrestre, na cidade de Oulu, Finlândia. Os autores avaliaram a taxa de perda de pacotes e a intensidade da potência do sinal recebido para as distâncias avaliadas.

Mikhaylov et al. [Mikhaylov et al., 2016], baseados nos resultados de [Petäjäjärvi et al., 2015], fazem uma análise do desempenho do LoRaWAN avaliando o rendimento em termos de taxa de transmissão, capacidade do canal para um número determinado de dispositivos terminais, e alcance entre os dispositivos terminais e os gateways. Os autores concluíram que o LoRaWAN, teoricamente, pode possuir milhões de nós conectados e enviando poucos bits, mas que só uma pequena porção desses dispositivos podem se localizar longe do gateway. Esta limitação é devida às interferências que podem afetar os enlaces (estruturas, outros sinais). Também concluíram que devido à limitação de ciclo de trabalho (duty cycle), o LoRaWAN pode reduzir a latência e o consumo de energia, apesar de ser altamente sensível a colisões de pacotes por conta do método de acesso ao meio (ALOHA).

Adelantado et al. [Adelantado et al., 2017] apresentam uma publicação na qual são avaliados os limites do LoRaWAN, com o objetivo de mostrar as limitações da tecnologia. Para isso, os autores discutem as capacidades e limitações da tecnologia no 
contexto de casos de uso. Baseado nas limitações do ciclo de trabalho, tempos de transmissão, tamanho do cabeçalho MAC (Media Access Control) e número de pacotes recebidos por hora, Adelantado et al. mostram que o número de pacotes recebidos por hora diminui conforme aumentam o número de nós, independentemente do tamanho do cabeçalho MAC ou da vazão dos dados úteis (goodput). Assim, os autores descrevem as aplicações que podem ser consideradas para a implementação de uma rede LoRa. Já Ortiz et al. [Ortiz et al., 2018] apresentam uma análise da tecnologia LoRa através de experimentos práticos, avaliando a capacidade da rede em termos de vazão, a taxa de perda de mensagens, a potência do sinal do enlace e a relação entre vazão teórica e a vazão obtida através dos experimentos. A análise dos experimentos foi feita em função da variação do fator de espalhamento. Assim, foi avaliada a eficácia do enlace para cada fator de espalhamento em um cenário com uma distância máxima de $2 \mathrm{~km}$. Os autores concluíram que o fator de espalhamento aumenta a sensibilidade no receptor, ao custo de menores taxas de transmissão.

O presente trabalho se diferencia dos descritos acima ao investigar a equivalência entre os resultados de simulações e experimentos práticos. Em vez de desenvolver uma nova forma de fazer simulação ou investigar detalhes da tecnologia, busca-se fornecer ao desenvolvedor de soluções que empreguem a comunicação via LoRa o conhecimento do quanto a simulação realmente espelha a realidade. Esta mesma informação pode ser usada para avaliar a simulação em si, contribuindo para o seu aperfeiçoamento e auxiliando pesquisadores a decidir pela sua utilização ou não. A seguir, é descrita brevemente a tecnologia LoRa e algumas das suas características principais.

\section{Tecnologia LoRa}

A LoRa é uma especificação de camada física (PHY) proprietária da Semtech [LoRa ${ }^{\mathrm{TM}}$-Alliance, 2016], desenhada para redes de longo alcance e baixa potência. Assim, esta tecnologia está projetada para permitir a conectividade de objetos inteligentes em distâncias da ordem dos quilômetros, com baixo consumo de energia, requerimento essencial das redes da Internet das Coisas.

A camada física (PHY) da tecnologia LoRa modula sinais em sub-bandas de rádio da faixa de frequências não licenciadas ISM (Industrial, Scientific and Medical) na ordem dos MHz. Para o Brasil, de acordo com a Agência Nacional de Telecomunicações (ANATEL), a faixa de frequências regulamentada para ISM está entre 915 e $928 \mathrm{MHz}$ (AU915-928MHz) [ANATEL, 2018]. A frequência de $433 \mathrm{MHz}$ (frequência de radioamador) pode ser implementada para redes com poucos dispositivos [ANATEL, 2016]. O LoRa PHY usa correção antecipada de erros (Forward Error Correction - FEC) e uma técnica de modulação de espalhamento espectral proprietária, que é uma variante do espalhamento espectral por chirp, que modula pulsos de chirps em frequência, a fim de codificar a informação [Bor et al., 2016a, LoRa ${ }^{\mathrm{TM}}$-Alliance, 2016].

\section{Metodologia dos Experimentos}

A metodologia adaptada para os experimentos define os critérios usados para configurar o modelo prático e o modelo simulado. A seguir, são expostos os parâmetros usados para avaliar as métricas propostas no artigo. 


\subsection{Parâmetros de Configuração do LoRa}

Os parâmetros de configuração disponíveis para a customização da camada física do LoRa são os seguintes: a frequência da portadora, a largura de banda, a taxa de código (Code Rate - CR), e o fator de espalhamento. A frequência da portadora define a frequência central para a banda de transmissão. É definida de acordo com a região de operação dos equipamentos. Assim sendo, este parâmetro não é, em geral, ajustável de acordo com a aplicação. A largura de banda, por sua vez, define o tamanho da faixa de frequências utilizada, com três valores programáveis: $125 \mathrm{kHz}, 250 \mathrm{kHz}$ e $500 \mathrm{kHz}$. O SF define o espalhamento espectral [Semtech Corp., 2013, Bor et al., 2016a]. Assim, estabelece a razão entre a taxa de bits e a taxa de chirps. A especificação LoRa define seis valores diferentes para o parâmetro fator de espalhamento: SF7, SF8, SF9, SF10, SF11 e SF12 [Semtech Corp., 2015], o que permite formar canais ortogonais, fazendo com que enlaces com fatores de espalhamento diferentes não tenham colisões entre si. Um maior SF aumenta a sensibilidade do limiar de recepção em termos de potência, porém, aumenta o tempo de propagação no ar e diminui a taxa de transmissão do enlace [Raza et al., 2017]. A taxa de bits, então, é dada por:

$$
R_{b}=S F \times \frac{B W}{2^{S F}}, \quad \operatorname{com} S F \in\{7,8,9,10,11,12\} .
$$

A CR define quantos bits são utilizados para redundância na mensagem, para realizar a recuperação de erros. A taxa de codificação $T_{c}$ [Semtech Corp., 2015] como:

$$
T_{c}=\frac{4}{4+C R}, \quad \operatorname{com} C R \in\{1,2,3,4\} .
$$

Também é especificada uma taxa de transmissão $R_{b}$ teórica, definida em função do fator de espalhamento e da taxa de código, representando a taxa de bits efetiva como:

$$
R_{b}=S F \times \frac{T_{c} \times B W}{2^{S F}}, \quad \operatorname{com} S F \in\{7,8,9,10,11,12\} .
$$

A Tabela 1 exibe os valores teóricos de taxa de transmissão de diferentes fatores de espalhamento, para uma CR igual a $4 / 5$ e uma largura de banda de $500 \mathrm{kHz}$. Esses valores teóricos são utilizados como referência para a análise de desempenho deste trabalho. A Seção 4 compara esses valores com valores obtidos experimentalmente, a fim de estabelecer bases para as aplicações que utilizam LoRa e LoRaWAN como tecnologias de comunicação.

Tabela 1. Valores teóricos de taxa de transmissão em função do SF.

\begin{tabular}{|c|c|}
\hline $\begin{array}{c}\text { Fator de } \\
\text { espalhamento }\end{array}$ & $\begin{array}{c}\text { Taxa de transmissão } \\
\text { teórica (bits/s) }\end{array}$ \\
\hline SF7 & 21875 \\
\hline SF8 & 12500 \\
\hline SF9 & 7031 \\
\hline SF10 & 3906 \\
\hline SF11 & 2148 \\
\hline SF12 & 1172 \\
\hline
\end{tabular}


Outro parâmetro necessário para descrever o comportamento do enlace de comunicação sem-fio na simulação é o modelo de propagação. Os modelos de propagação descrevem o comportamento das ondas eletromagnéticas, e como elas são irradiadas desde o transmissor até o receptor. O modelo de propagação que descreve melhor o cenário escolhido para os experimentos práticos é o LogDistance. Este modelo é amplamente usado em cenários de larga escala e em enlaces com frequências da ordem dos $\mathrm{MHz}$, e prediz a atenuação do sinal, perto dos valores que foram medidos nos experimentos práticos, usando como parâmetros a potência do sinal e a distância:

$$
P L(d)=P L\left(d_{o}\right)+10 n \log \left(\frac{d}{d_{o}}\right)
$$

Devido ao LogDistance implementado no simulador ser um modelo que não considera a variação da atenuação por flutuações do meio de propagação, foi adaptada uma variável de distribuição normal randômica que descreve as perdas ocasionadas pelo fenômeno de desvanecimento [Rappaport, 2001]. Assim, o modelo de propagação usado para a comparação entre os experimentos práticos e simulados foi implementado usando a seguinte equação:

$$
P L(d)=P L\left(d_{o}\right)+10 n \log \left(\frac{d}{d_{o}}\right)+X_{\sigma},
$$

onde $n$ descreve o coeficiente empírico de propagação, e $X_{\sigma}$ descreve a variação do desvanecimento em termos de potência $(\mathrm{dB})$; o intervalo de valores para $X_{\sigma}$ foi obtido a partir dos dados obtidos da potência do sinal nos experimentos práticos.

\subsection{Parâmetros dos Experimentos Desenvolvidos}

Os experimentos realizados para a avaliação da tecnologia LoRa consistem de medições práticas e simuladas da vazão, da taxa de perda, da potência do sinal e da relação entre a vazão teórica e a vazão obtida através dos experimentos. Todas as métricas são avaliadas em função da variação do fator de espalhamento e da distância. As medições práticas são realizadas por meio da implementação de um protótipo em hardware, enquanto as simulações são executadas utilizando a biblioteca LoRaWAN implementada em [Magrin et al., 2017] para o simulador de rede NS-3.

Os parâmetros de configuração dos módulos são descritos na Tabela 2. A comunicação consiste na transmissão de tuplas de dados pela unidade transmissora para cada valor de SF $(7,8,9,10,11)$ programado. O SF12 não está disponível na biblioteca usada. Para cada transmissão, são feitas 10 rodadas, e em cada rodada são transmitidos 51 pacotes, numerados de 0 a 50. Cada pacote inclui o número de sequência, as coordenadas geográficas (obtidas pelo receptor GPS a cada $250 \mathrm{~ms}$ ), a altitude, a temperatura e a umidade (ambas medidas pelo sensor). Foram usados 64 bits de preâmbulo. $\mathrm{O}$ tamanho total do pacote enviado é de 47 bytes. No cenário escolhido, é feito um estudo comparativo do comportamento dos fatores de espalhamento definidos na camada física do LoRa em função da distância.

Para realizar os experimentos práticos com os dispositivos físicos LoRa, é implementado um protótipo em hardware proposto em [Ortiz et al., 2018]. As simulações são 
Tabela 2. Parâmetros de configuração dos experimentos práticos.

\begin{tabular}{|c|c|}
\hline Frequência & $915 \mathrm{MHz}$ \\
\hline Potência de transmissão & $14 \mathrm{dBm}$ \\
\hline Fator de espalhamento & $\mathrm{SF}(7,8,9,10,11)$ \\
\hline Largura de banda & $500 \mathrm{kHz}$ \\
\hline Taxa de código & $4 / 5$ \\
\hline
\end{tabular}

feitas no NS-3, na versão 28. É utilizada e adaptada a biblioteca pública do módulo de rede LoRaWAN disponível $\mathrm{em}^{1}$ [Magrin et al., 2017], que simula até a camada de rede. Os modelos de implementação fornecidos são ajustados para compatibilizá-los com os cenários de experimentação planejados para este trabalho. Para isso, é gerado um ambiente de simulação em que os nós se comunicam às distâncias estabelecidas no experimento prático (Seção 4.3). É utilizada na simulação a mesma metodologia de envio de pacotes em função da variação do SF e da distância, assim como os parâmetros mostrados na Tabela 2. É utilizado o modelo de propagação LogDistance nativo do NS-3, que se aplica à natureza do ambiente dos experimentos práticos, área urbana com linha de visada desobstruída. Entre os modelos avaliados, o LogDistance é o que mais se aproxima do comportamento observado nos experimentos práticos. Entretanto, para descrever melhor a variação do sinal, é acrescentada neste trabalho uma variável de distribuição normal randômica com média $\mu=0$ e $\sigma^{2}=1,47$, descrita na Equação 5. Estes valores que descrevem a variável $X_{\sigma}$ foram obtidos a partir dos experimentos práticos.

\subsection{Cenário dos Experimentos}

O cenário utilizado para a realização dos experimentos para avaliação prática da tecnologia LoRa compreende pontos localizados na Ilha do Governador e no campus da Universidade Federal do Rio de Janeiro (UFRJ), Ilha do Fundão, selecionados de forma a obter enlaces com linha de visada desobstruída e comprimento variável. Foram usadas duas locações para a unidade receptora: o ponto mais alto do edifício do Centro de Tecnologia e do edifício do Hospital Universitário Clementino Fraga Filho (HUCFF/UFRJ). Já a unidade transmissora foi posicionada em seis pontos pré-definidos a 0, 1000, 2000, 3000, 4000 e $5000 \mathrm{~m}$ de distância. A Figura 1 mostra o local dos experimentos.

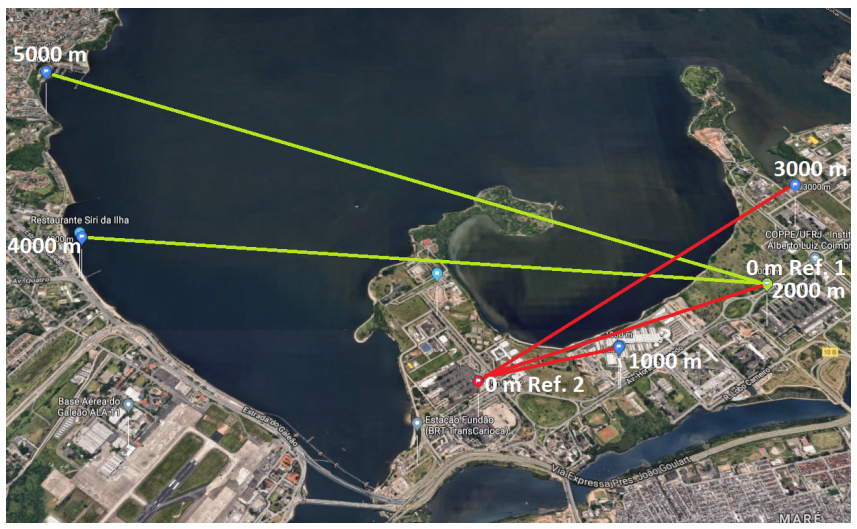

Figura 1. Visão aérea da llha do Governador e do campus llha do Fundão da UFRJ, cenário dos experimentos práticos (obtida com o Google Earth).

\footnotetext{
${ }^{1}$ https://github.com/signetlabdei/lorawan
} 


\section{Resultados}

A partir das configurações implementadas nos experimentos práticos e simulados, descritos na Seção 4, são comparados os resultados das métricas de vazão, taxa de perda, potência do sinal e a relação entre a vazão teórica e a vazão obtida dos experimentos. Em todos os cenários, foram feitas 10 rodadas para cada distância e fator de espalhamento, com intervalo de confiança de $95 \%$, representado por barras de erro verticais nos gráficos. Para determinar a correlação dos experimentos práticos e simulados, foi usada a análise correlacional de Pearson, mostrada na Equação 6.

$$
r=\frac{\Sigma\left(x-\mu_{x}\right)\left(y-\mu_{y}\right)}{\sqrt{\Sigma\left(x-\mu_{x}\right)^{2} \Sigma\left(y-\mu_{y}\right)^{2}}}
$$

\subsection{Análise de Vazão}

Para avaliar a vazão, são considerados os tempos entre a primeira e a última recepção do conjunto de pacotes enviado, assim como o tamanho de cada pacote enviado. As figuras 2(a) e 2(b) apresentam a vazão do enlace em segmentos de $1000 \mathrm{~m}$ nos experimentos práticos e simulados. Como pode-se observar, nos experimentos práticos e simulados todos os SFs mantiveram uma vazão constante para todos os segmentos, até a qualidade do enlace se degradar pelo aumento da distância, diminuindo a vazão. Esse comportamento está de acordo com o esperado para distâncias maiores e é percebido de forma mais acentuada nos SF7 e SF8. A vazão máxima experimental foi de $\approx 1 \mathrm{kbit} / \mathrm{s}$, semelhante ao experimento simulado. Também se observa que no SF7 e SF8 a degradação do sinal aconteceu um segmento antes que no experimento prático. Isto está associado aos níveis de sensibilidade de recepção. No modelo simulado, há um nível de sensibilidade fixo para cada SF. No modelo prático, há uma faixa de sensibilidade informada para cada SF [Dragino, 2017]. A diferença entre os valores de sensibilidade real e simulado influenciou mais os resultados para o SF7 e SF8.

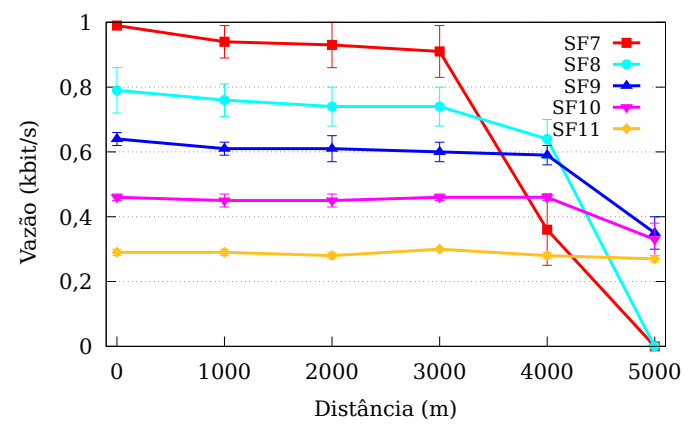

(a) Vazão (experimento prático).

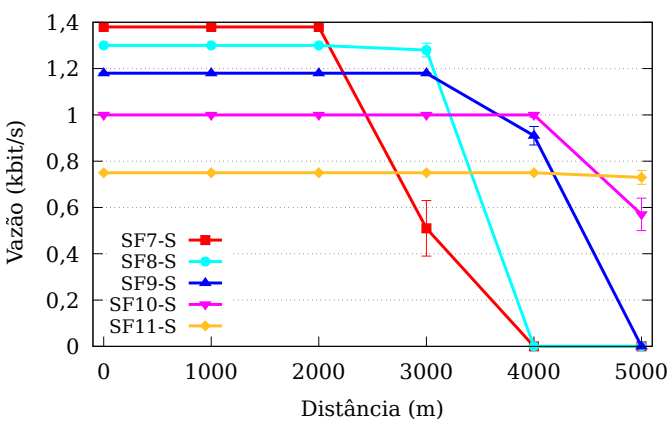

(b) Vazão (simulação).

Figura 2. Vazão obtida nos experimentos prático e simulado.

Tabela 3. Correlação da vazão nos experimentos prático/simulado.
\begin{tabular}{|c|c|c|c|c|c|}
\hline SF7/SF7-S & SF8/SF8-S & SF9/SF9-S & SF10/SF10-S & SF11/SF11-S \\
\hline$r$ & 0,8568606 & 0,7484335 & 0,9829023 & 0,9954955 & 0,9079594 \\
\hline
\end{tabular}

No entanto, o resultado das curvas obtidas nos experimentos práticos e simulados correspondem ao comportamento teórico da tecnologia, como é mostrado na Tabela 3 , 
onde é apresentada a correlação entre a vazão prática e a simulada. É possível concluir que qualquer variação na vazão prática está representada na vazão simulada, mostrando um grau de associação alto nos experimentos, com excepção no SF7-S e o SF8-S, que apresenta uma correlação moderada com os outros SFs por conta da diferença na vazão. Isto quer dizer que qualquer variação no experimento prático será refletida no experimento simulado, sempre e quando o cenário for o mesmo.

\subsection{Análise da Taxa de Perda}

Para a análise da taxa de perda, são considerados os números de sequência que identificam cada pacote no experimento prático. A taxa de perda observada nos experimentos prático/simulado apresenta um comportamento condizente com os resultados da análise da vazão, aumentando com a distância. As Figuras 3(a) e 3(b) apresentam a taxa de perda no enlace nos experimentos práticos e simulados, respectivamente. Comparando as curvas obtidas, a taxa de perda apresenta comportamento semelhante na prática e simulação. No experimento simulado, a perda de pacotes nos SF7 e SF8 inicia $1000 \mathrm{~m}$ antes em comparação com o experimento prático. Por outro lado, o SF9 teve uma taxa de perda total em $5000 \mathrm{~m}$, enquanto no experimento prático teve uma perda inferior a $20 \%$. Já o SF11 teve um comportamento semelhante ao SF11 do experimento prático, exceto no ponto de $1000 \mathrm{~m}$, onde teve uma perda de $\approx 10 \%$. Esta perda se deve a uma maior atenuação causada pela presença de árvores no enlace. Embora não obstruam a linha de visada, o cenário dos experimentos apresenta variações de ambiente entre os diferentes pontos. Por fim, ao analisar o SF10 e SF11, verifica-se uma perda não superior a $20 \%$ nos experimentos práticos, e uma perda não superior a 50\% nos experimentos simulados, o que indica que em condições ideais de linha de visada, o enlace pode atingir distâncias maiores usando os fatores de espalhamento mais altos.

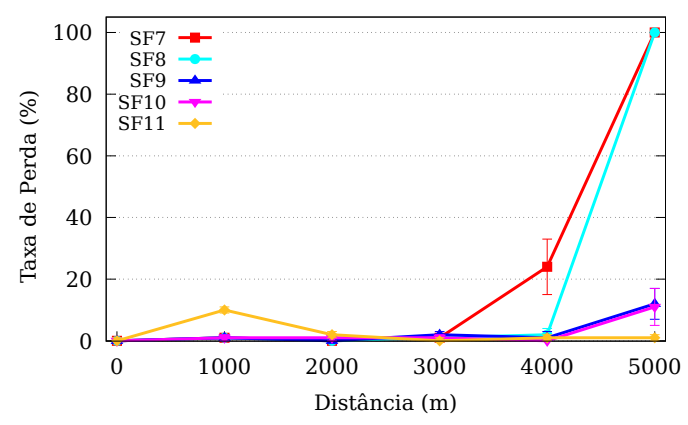

(a) Taxa de perda (experimento prático).

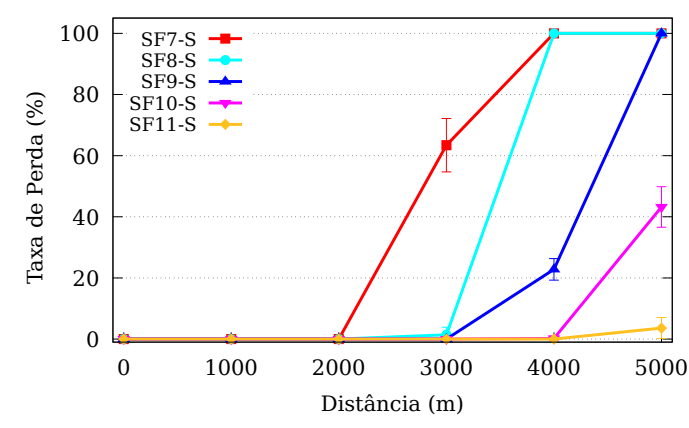

(b) Taxa de perda (simulação).

Figura 3. Taxa de perda obtida nos experimentos prático e simulado.

Tabela 4. Correlação da taxa de perda nos experimentos prático/simulado.

\begin{tabular}{|c|c|c|c|c|c|}
\cline { 2 - 6 } \multicolumn{1}{c|}{} & SF7/SF7-S & SF8/SF8-S & SF9/SF9-S & SF10/SF10-S & SF11/SF11-S \\
\hline$r$ & 0,6973851 & 0,6438531 & 0,9658178 & 0,9930800 & $-0,1705606$ \\
\hline
\end{tabular}

A correlação entre os resultados da taxa de perda é mostrada na Tabela 4. Se observa que há uma correlação com grau de associação negativo entre o SF11 e os SFs simulados. Isto acontece porque, embora na prática o SF11 consiga manter a comunicação do enlace em $5000 \mathrm{~m}$, teve perdas recorrentes em todos os pontos, além de uma perda 
acentuada no ponto de $1000 \mathrm{~m}$, o que altera a correlação com os outros SFs. Diferente do prático, no experimento simulado, enquanto houve troca de pacotes, a perda foi diferente de 0 para os SF maiores.

\subsection{Medições de Potência do Sinal}

As medições de potência do sinal foram obtidas das bibliotecas implementadas nos experimentos práticos e nos experimentos simulados, não se utilizando um instrumento de medidas externo. As Figuras 4(a) e 4(b) apresentam a potência do sinal no enlace. O comportamento é muito semelhante para os dois experimentos, apesar da diferença inicial de $\approx-10 \mathrm{dBm}$. Assim, em $0 \mathrm{~m}$, a potência do sinal no ambiente simulado foi de $\approx-50 \mathrm{dBm}$, enquanto no experimento prático foi de $\approx-37 \mathrm{dBm}$. Já em $1000 \mathrm{~m}$, é medida a mesma potência $(\approx-110 \mathrm{dBm})$ para os dois experimentos. Nas distâncias seguintes a potência reduz exponencialmente. $\mathrm{O}$ experimento prático apresenta algumas variações de potência devido ao cenário onde foi implementado, mas sem diferença estatística representativa. Se observa essa variação nos pontos de $2000 \mathrm{~m}$ e $3000 \mathrm{~m}$. Já nos pontos de $4000 \mathrm{~m}$ e $5000 \mathrm{~m}$, a tendência da potência foi semelhante, decrescendo abaixo dos $-120 \mathrm{dBm}$. Nos dois experimentos, há uma queda abrupta nos primeiros $1000 \mathrm{~m}$, e mantendo um decremento exponencial com o aumento da distância. No experimento simulado a potência do sinal vai decrescendo exponencialmente, conforme o descrito na literatura. Nos experimentos práticos, o único SF que mostrou um comportamento anormal foi o SF7 no ponto de $4000 \mathrm{~m}$, onde mediu-se uma melhora na potência do sinal, com respeito aos pontos anteriores. Isso é atribuído a variações na atenuação do enlace e na propagação da onda em si. Uma vez que para atingir este ponto o enlace passa por um espelho de água, que pode eventualmente causar uma composição construtiva entre os raios direto e refletido.

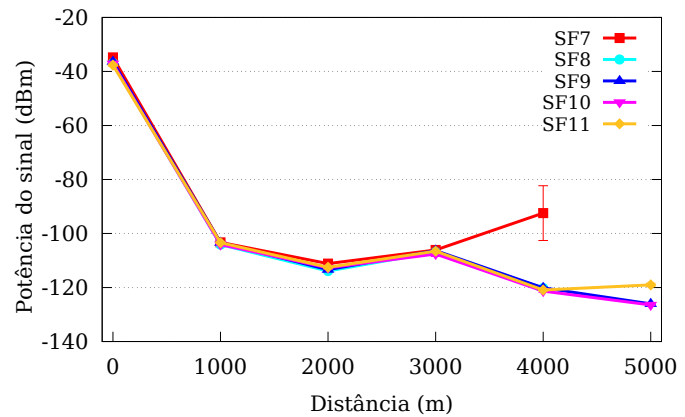

(a) Potência do sinal (experimento prático).

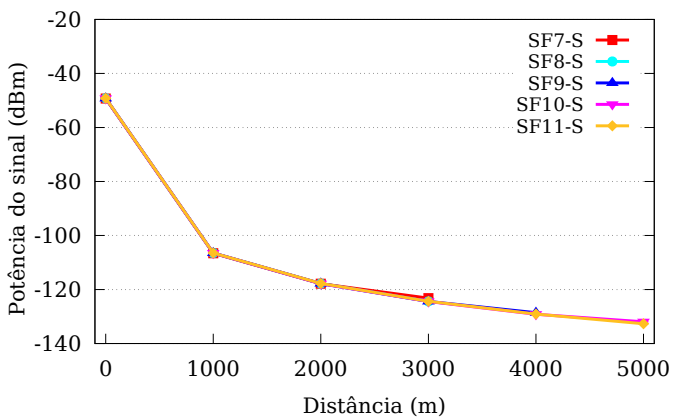

(b) Potência do sinal (simulação).

Figura 4. Potência do sinal obtida nos experimentos prático e simulado.

Tabela 5. Correlação da potência do sinal nos experimentos prático/simulado.

\begin{tabular}{|c|c|c|c|c|c|}
\cline { 2 - 6 } \multicolumn{1}{c|}{} & SF7/SF7-S & SF8/SF8-S & SF9/SF9-S & SF10/SF10-S & SF11/SF11-S \\
\hline$r$ & 0,8457486 & 0,9575271 & 0,9748970 & 0,9874096 & 0,9845240 \\
\hline
\end{tabular}

Condizente com os resultados dos experimentos prático/simulado, todos fatores de espalhamento estão fortemente correlacionados. A Tabela 5 mostra que os resultados de potência do sinal dos experimentos prático e simulado correspondem um com o outro. 


\subsection{Relação de Vazão Teórica vs. Vazão Prática}

Para avaliar a capacidade do enlace, foi comparada a taxa de transmissão calculada na Tabela 1, e os valores obtidos nos experimentos prático e simulado. As Figuras 5(a) e 5(b) mostram o resultado da relação entre vazão teórica e vazão medida. Enquanto no experimento prático a relação varia de $4 \%$ a $14 \%$, no experimento simulado a relação assume valores de $6 \%$ a $36 \%$. Este resultado mostra que no SF7 há uma relação similar, com a relação em torno de $5 \%$ para os experimentos prático e simulado. Nos outros SFs, o experimento simulado apresenta valores que praticamente dobram a relação de vazão teórica versus prática em comparação com os valores obtidos no experimento prático. Estes resultados estão associados ao atraso inserido para obter dados de localização atualizados (de $250 \mathrm{~ms}$ ). Em [Ortiz et al., 2018], a relação de vazão foi maior, porém, foram enviadas tuplas de dados com bits aleatórios para atingir a máxima capacidade do enlace, o que incorreu em uma taxa de perda maior em menores distâncias, e menor alcance.

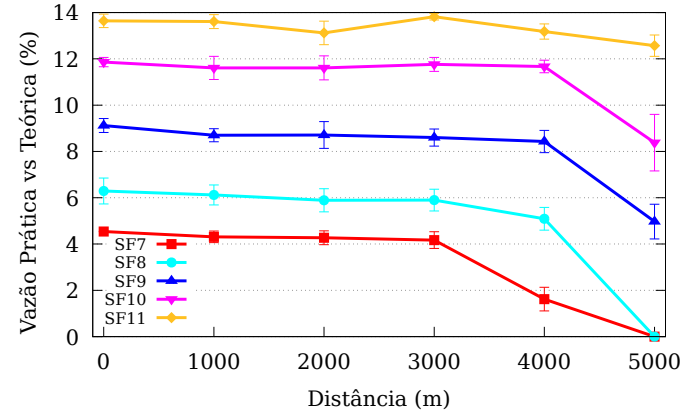

(a) Vazão prática vs. vazão teórica (experimento prático).

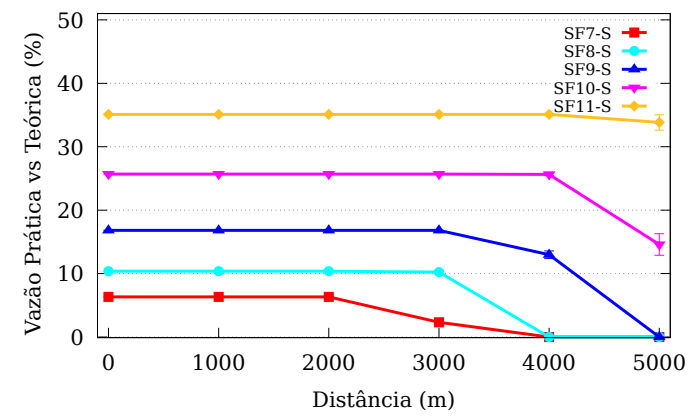

(b) Vazão prática $v s$. vazão teórica (simulação).

Figura 5. Vazão prática vs. vazão teórica nos experimentos prático e simulado.

Os resultados dos experimentos demonstram, como esperado, o comportamento da camada física do LoRa em um enlace. A influência do tamanho do pacote torna-se importante porque o enlace é altamente suscetível a interferências e, portanto, há erros de bit que podem piorar o desempenho da rede na presença de mais dispositivos terminais. No experimento prático, a redução do tamanho do pacote permitiu ter um maior alcance entre a unidade transmissora e receptora, chegando neste trabalho a $5000 \mathrm{~m}$. Por outro lado, devido que a transmissão está sendo espalhada através de diferentes ambientes (vegetação, espelhos de água, variações climáticas), há a tendência de que a qualidade da comunicação do enlace torne-se instável e com fortes variações de potência de sinal e perda de pacotes. Como foi observado na Seção 5.3, as medições de $4000 \mathrm{~m}$ e $5000 \mathrm{~m}$, que ocorrem acima da água, apresentam variações na potência do sinal, especificamente nos SF7, SF10 e SF11. O SF11 também apresenta alterações nas medições de $1000 \mathrm{~m}$ por conta da vegetação ao redor da antena transmissora, como foi observado na Seção 5.2. Portanto percebe-se que a simulação de redes de longo alcance torna-se fundamental para o planejamento de redes LPWAN, para que seja possível avaliar os enlaces sem estar sujeito a estas diferenças do ambiente. Por isto, é imperativo que o modelo de simulação da camada física do LoRa, projetado para o simulador NS-3, continue sendo aprimorado, de forma a refletir um ambiente mais coerente com aquele obtido com o experimento prático. 


\section{Conclusão e Trabalhos Futuros}

Este trabalho comparou a avaliação de desempenho de uma rede LoRa através de experimentos práticos e simulados. O objetivo foi analisar a correspondência entre os dados coletados nos dois ambientes. Foi utilizado para a simulação um modelo de camada física LoRa implementado no simulador NS-3. Dentro deste modelo, foi utilizado um modelo de propagação adequado para grandes áreas, e calculada uma variável para a análise das perdas por desvanecimento do sinal, característico das redes LPWAN. Os resultados revelaram que todas as métricas avaliadas nos experimentos simulados foram consistentes com os resultados dos experimentos práticos. Por outro lado, as diferenças encontradas nos resultados dos experimentos práticos e simulados sugerem um aperfeiçoamento no modelo usado para a simulação do enlace LoRa. Pode-se concluir que o modelo de simulação pode ser melhorado, procurando uma correlação mais forte com experimentos práticos.

Como trabalhos futuros, a operação da biblioteca do NS-3 lorawan será analisada no contexto de redes de múltiplos saltos, avaliação da vazão máxima da rede, quantidade de dispositivos conectados e testes de dispositivos classe B e C. Também pretende-se avaliar a combinação com outras tecnologias para IoT, a fim de aproveitar as vantagens de cada uma em termos de alcance, consumo de energia e taxas de dados.

\section{Agradecimentos}

Este trabalho foi parcialmente financiado pelas agências de fomento CAPES, CNPq, FAPERJ e pelos processos n ${ }^{0}$ 15/24494-8 e n n $^{\circ}$ 15/24490-2, da Fundação de Amparo à Pesquisa do Estado de São Paulo (FAPESP).

\section{Referências}

Adelantado, F., Vilajosana, X., Tuset-Peiro, P., Martinez, B., Melia-Segui, J. e Watteyne, T. (2017). Understanding the limits of LoRaWAN. IEEE Communications Magazine, 55(9):34-40.

ANATEL (2016). Plano de atribuição, destinação e distribuição de frequências no Brasil. http://www anatel.gov.br/institucional/acervo-documental. Acessado em fevereiro de 2019.

ANATEL (2018). Faixas de radiofrequências utilizáveis por equipamentos de radiação restrita. http://www.anatel.gov.br/legislacao/resolucoes/ 2018/1220-resolucao-705. Acessado em fevereiro de 2019.

Augustin, A., Yi, J., Clausen, T. e Townsley, W. M. (2016). A study of LoRa: Long range $\&$ low power networks for the Internet of things. Sensors, 16(9):1466.

Bor, M., Vidler, J. e Roedig, U. (2016a). Lora for the internet of things. Em International Conference on Embedded Wireless Systems and Networks (EWSN), p. 361-366.

Bor, M. C., Roedig, U., Voigt, T. e Alonso, J. M. (2016b). Do LoRa low-power widearea networks scale? Em ACM International Conference on Modeling, Analysis and Simulation of Wireless and Mobile Systems (MSWiM), p. 59-67.

Carneiro, G. (2010). NS-3: Network simulator 3. Em UTM Lab Meeting April, volume 20. 
Centenaro, M., Vangelista, L., Zanella, A. e Zorzi, M. (2016). Long-range communications in unlicensed bands: the rising stars in the IoT and smart city scenarios. IEEE Wireless Communications, 23(5):60-67.

Dragino (2017). Manual. Disponível em: http://wiki.dragino.com/index. php?title=Lora \_Shield. Acessado em fevereiro de 2019.

Evans, D. (2011). The internet of things: How the next evolution of the Internet is changing everything. Cisco Internet Business Solutions Group (IBSG), 1:1-11.

Goursaud, C. e Gorce, J.-M. (2015). Dedicated networks for IoT : PHY / MAC state of the art and challenges. EAI endorsed transactions on Internet of Things.

Henderson, T. R., Lacage, M., Riley, G. F., Dowell, C. e Kopena, J. (2008). Network simulations with the NS-3 simulator. Em SIGCOMM Demo, p. 527.

LoRa ${ }^{\mathrm{TM}}$-Alliance (2016). LoRa ${ }^{\mathrm{TM}}$-alliance technology. Disponível em: https: // www. lora-alliance. org/technology. Acessado em fevereiro de 2019.

Magrin, D., Centenaro, M. e Vangelista, L. (2017). Performance evaluation of LoRa networks in a smart city scenario. Em IEEE International Conference on Communications (ICC), p. 1-7.

Mikhaylov, K., Petäjäjärvi, J. e Haenninen, T. (2016). Analysis of capacity and scalability of the LoRa low power wide area network technology. Em European Wireless Conference, p. 1-6.

Ortiz, F. M., Cruz, P., de S. Couto, R. e Costa, L. H. M. K. (2018). Caracterização de uma Rede Sem-fio de Baixa Potência e Longo Alcance para Internet das Coisas. Em Simpósio Brasileiro de Redes de Computadores (SBRC).

Petäjäjärvi, J., Mikhaylov, K., Roivainen, A., Hanninen, T. e Pettissalo, M. (2015). On the coverage of LPWANs: range evaluation and channel attenuation model for LoRa technology. Em International Conference on ITS Telecommunications (ITST), p. 5559.

Rappaport, T. (2001). Wireless Communications: Principles and Practice. Prentice Hall PTR, Upper Saddle River, NJ, USA, 2a edição.

Raza, U., Kulkarni, P. e Sooriyabandara, M. (2017). Low power wide area networks: An overview. IEEE Communications Surveys \& Tutorials, 19(2):855-873.

Reynders, B., Meert, W. e Pollin, S. (2016). Range and coexistence analysis of long range unlicensed communication. Em International Conference on Telecommunications (ICT), p. 1-6.

Semtech Corp. (2013). LoRa ${ }^{\mathrm{TM}}$ modem designer's guide. Disponível em: https: // www. semtech. com/uploads / documents/. Acessado em fevereiro de 2019.

Semtech Corp. (2015). Lora ${ }^{\mathrm{TM}}$ modulation basics an1200.22. Disponível em: https : / / www. semtech. com/uploads / documents/. Acessado em fevereiro de 2019.

Van den Abeele, F., Haxhibeqiri, J., Moerman, I. e Hoebeke, J. (2017). Scalability analysis of large-scale LoRaWAN networks in NS-3. IEEE Internet of Things Journal, 4(6):2186-2198. 